\title{
Curcumin sensitizes response to cytarabine in acute myeloid leukemia by regulating intestinal microbiota
}

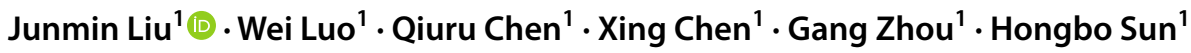

Received: 27 August 2021 / Accepted: 10 December 2021 / Published online: 23 January 2022

(c) The Author(s) 2021

\begin{abstract}
Purpose To address whether Curcumin has synergistic effect with cytarabine (Ara-C) in treating acute myeloid leukemia (AML).

Methods A xenograft AML mouse model was established by injecting HL-60 cells into tail vein of mice to assess the function of Curcumin. Mononuclear cells (MNCs) isolated from AML mice and AML cell lines were used to examine the effect of Curcumin. Metagenomics and metabolomics were used to evaluate the alteration of intestinal microbiota and the change of metabolites in MNCs.

Results Curcumin treatment sensitized response to Ara-C in MNCs of AML mice, but had no direct effect on AML cell lines. Metagenomics revealed an alteration of intestinal microbiota with Curcumin treatment, which contributes to sensitized response to Ara-C. Curcumin treatment led to enhanced intestinal intact to sensitize response to Ara-C in AML mice, through reducing mucus degrading bacteria. Metabolomics demonstrated that Curcumin treatment led to decreased cholesterol in MNCs of AML mice. Further study proved that Curcumin treatment resulted in inhibition of SQLE, a key enzyme of cholesterol biosynthesis, to increase sensitivity to Ara-C.

Conclusion Curcumin sensitizes response to Ara-C through regulating microbiota, highlighting the importance of intestinal intact strengthening in chemoresistant therapy. Moreover, aiming at cholesterol synthesis is promising in AML treatment.
\end{abstract}

Keywords Curcumin $\cdot$ Cytarabine $\cdot$ Acute myeloid leukemia $\cdot$ Microbiota $\cdot$ Cholesterol $\cdot$ SQLE

\section{Introduction}

Acute myeloid leukemia (AML) originates from the differentiation block of myeloid cells in the bone marrow (BM) [1]. As a malignant tumor of the blood system, the survival rate of AML is low $(<40 \%)$, which is mainly due to chemoresistance [2]. Despite a high rate of complete remission after treatment with chemotherapy drugs, the relapse rate remains very high and the prognosis very poor [1]. Therefore, ameliorating chemoresistance remains to be a clinical challenge.

\section{Junmin Liu}

liujunmin0614@126.com

1 Department of Hematology, Affiliated Hospital of Southern Medical University, Shenzhen Longhua District People's Hospital, No. 38 Jinglong Jianshe Road, Longhua District, Guangdong Province 518109 Shenzhen, People's Republic of China
Curcumin is a natural phenolic compound extracted from curcuma longa, which exerts a wide range of biological effects, such as anti-tumor, anti-inflammatory, anti-oxidation and anti-fibrosis [3, 4]. Moreover, Curcumin was also reported to affect energy metabolism to increase energy expenditure as well as improve insulin sensitivity in obese mice [5, 6]. Energy metabolism also plays crucial roles in response to chemotherapy. Several studies have reported that targeting energy metabolism could sensitize resistant cells to chemotherapy, such as oxidative phosphorylation inhibitor redirected metabolism toward glycolysis to sensitize resistant cells to cytarabine (Ara-C) in AML [7]. On the other hand, Curcumin could affect intestinal microbiota to prevent renal failure [6]. Conversely, intestinal microbiota could also enhance the effect of Curcumin in ameliorating HFDinduced obesity by enhancing Ucp1-dependent thermogenesis through regulating bile acids metabolism [8], suggesting the interaction between intestinal microbiota and Curcumin in curing diseases. 
In the present study, we will investigate the synergistic effect between Curcumin and Ara-C using AML mouse xenograft mouse model and its potential mechanism.

\section{Materials and methods}

\section{Xenotransplantation of human leukemic cells}

Xenograft AML model was established using a regular method as previously described $[9,10]$. Briefly, Cultured HL-60 cell line was washed twice in phosphate-buffered saline (PBS) and cleared aggregates and debris using a $0.2-\mathrm{mm}$ cell filter, which were suspended in PBS at a final concentration of 2 million cells per $200 \mu 1$ of PBS per mouse for intravenous injection. As the molecular changes is different between male and female patients, we used male mice for the model establishment. Xenograft tumors were generated by injecting AML cells (in $200 \mu \mathrm{l}$ of PBS) in the tail vein of 6-week-old male NSG mice. Daily monitoring of mice for symptoms of disease (ruffled coat, hunched back, weakness and reduced motility) determined the time of killing for injected animals with signs of distress. Mice were raised in the animal room of Shnezhen Longhua New District People's Hospital. Germ free mice were obtained from Cyagen and raised in Germ free package. The committee of Shnezhen Longhua New District People's Hospital approved this study. All animal experiments comply with the ARRIVE guidelines and carried out in accordance with the National Institutes of Health guide for the care and use of Laboratory animals (NIH Publications No. 8023, revised 1978).

\section{Cytarabine, curcumin, VSL\#3 and terbinafine treatment}

Following the mice engraftment (symptoms of disease at week 3 after cell injection), we started to treat them with daily intraperitoneal injections of $30 \mathrm{mg} / \mathrm{kg}$ Ara-C for 5 days [10]. The in vivo experiments were performed in NSG recipients transplanted. For negative control, NSG mice were treated daily with PBS for 5 days. Mice were monitored for toxicity and provided with nutritional supplements as needed. Curcumin was proved to be nontoxic and it was administrated to mice $(200 \mathrm{mg} / \mathrm{kg}$ body weight daily) intraperitoneally when Ara-C was treated. VSL\#3 (15 mg, mixture of Lactobacilli, Bifidobacteria and Streptococcus) was orally administrated to mice daily one week before Ara-C was treated. Terbinafine was also orally administrated to mice ( $80 \mathrm{mg} / \mathrm{kg}$ body weight daily) when Ara-C was treated.

\section{MNCs isolation and cell culture}

MNCs were isolated from mice with a standard Ficoll-Hypaque gradient separation method as previously used [11]. The committee of Shenzhen Longhua New District People's Hospital approves this experiment. MNCs HL-60 and THP-1 (obtained from ATCC) were cultured in RPMI-1640 medium (Gibco; Thermo Fisher Scientific, Inc.) supplemented with $10 \% \mathrm{FBS}, 100 \mathrm{U} / \mathrm{mL}$ penicillin, and $100 \mathrm{mg} / \mathrm{mL}$ streptomycin at $37^{\circ} \mathrm{C}$ in a humidified atmosphere containing $5 \% \mathrm{CO} 2$.

\section{Western blotting}

Protein was extracted from cells using cold RIPA buffer with protease inhibitors and phosphatase inhibitor. Then, protein was separated in $10 \%$ SDS-PAGE and transferred into nitrocellulose membrane (NC). After blocking for $30 \mathrm{~min}$ with $1 \% \mathrm{BSA}$, the membranes were incubated with primary antibodies at $4{ }^{\circ} \mathrm{C}$ overnight. Then, membrane was incubated with secondary antibodies for $1 \mathrm{~h}$ at room temperature. Finally, protein was detected using ChemiDoc MP Imaging System.

\section{Intestine permeability assay}

To determine intestinal permeability, mice were starved overnight, followed by gavaging FITC-dextran (Sigma \#FD4) orally (44 mg/100 g body weight). After $4 \mathrm{~h}$, mice were anesthetized, then blood was collected by cardiac puncture using $1 \mathrm{ml}$ syringe and finally mice were killed. Serum was separated using Tubes containing coagulant (BD $\# 365,968)$ and diluted with an equal volume of PBS. $100 \mu 1$ of diluted serum was added to a 96-well microplate [12]. The concentration of FITC in serum was determined by spectrophotofluorometry (BioTek).

\section{SQLE over-expression}

pRL-cyto-megalovirus (pCMV)-SQLE (RC202008) and pCMV-entry control plasmids (OriGene) were transfected into cells using Lipofectamine 2000 (Invitrogen) according to the manufacturer's instructions.

\section{Cell viability and apoptosis assay}

AML cells were seeded into 96-well plates (500 cells/ well). Then, cells were incubated with drugs [(Ara-C HL-60:0.1 $\mu \mathrm{M}$; THP-3 $\mu \mathrm{M}$ ), Curcumin (serial concentrations)]. CCK-8 reagent ( $10 \mu \mathrm{L})$ were added to 96 -well plates, 
incubating for another $1 \mathrm{~h}$ at $37{ }^{\circ} \mathrm{C}$ in dark. The absorbance was studied at an OD of $450 \mathrm{~nm}$ by a microplate reader. Cell proliferation rate $(\%)=\mathrm{OD}$ value of experimental well/ OD value of blank well $\times 100 \%$. Apoptosis was evaluated using an annexin-phycoerythrin/7-aminoactinomycin D staining kit (BD Biosciences).

\section{Cholesterol concentration}

MNCs (106) were harvested, and cholesterol concentration was assessed by Cholesterol Quantification kit (ab65359, Abcam) according to the manufacturer's instruction.

\section{Shot-gun metagenome sequencing and analyses}

Stool was collected from Cur/A- and Ara-C-treated AML mice. DNA of stool was extracted using PowerFecal DNA isolation kit (QIAGEN) according to the manufacturer's instruction. DNA concentration was measured using NanoDrop. Amplified library was generated using Era XT adapters. HiSeq Illuminex 2500 was used for sequencing, which was performed to obtain DNA (125 bp) paired end reads to a depth of $10 \mathrm{G}$ base pairs per sample. For sequencing data, BWA and SAM tools were used for host DNA removal and quality control as previously described $[13,14]$. Then, Kraken 2 and Bracken were applied for species-level taxonomic profiling using high-quality reads [16]. Bacteria species with adjusted $p$-value $<0.05$ and $\mid \log 2$ (fold change) $\mid>1$ were considered statistically significant.

\section{Metabolomics profiling and analyses}

Metabolomics was performed in the company of Novogene. Briefly, MNCs (106) was homogenized on ice and dissolved in $500 \mathrm{uL}$ of cold water. Then, samples were vortexed and centrifuged $(10,000 \mathrm{~g})$ for $15 \mathrm{~min}$. Supernatant was collected and the pellets were further extracted using $500 \mathrm{uL}$ of cold methanol. Cells' extracts were mixed and centrifuged with $10,000 \mathrm{~g}$ for $15 \mathrm{~min}$. Supernatant was used for LC-MS analysis. A waters Acquity Ultra-high-Performance LC system, equipped with a BEHC18 column (Milford, USA), was applied using chromatographic analysis. The mobile phases consisted of solvents A and B. The elution gradient program for MNCs was: $0-1 \%$ B for $0.5 \mathrm{~min} ; 1-30 \% \mathrm{~B}$ from 0.5 to $5 \mathrm{~min} ; 30-50 \%$ B from 5 to $13 \mathrm{~min}$ and $50-100 \%$ B from 13 to $17 \mathrm{~min}$; the flow rate was $0.45 \mathrm{ml} / \mathrm{min}$. Mass spectra were obtained from a Waters SYNAPT G2 HDMS (Waters Corp., Manchester, UK) TOF mass spectrometer combined with an ESI source with positive ion scan mode.

The metabolomic data were analyzed using Metabo AnalystR. Wilcoxon rank-sum test was used to determined significantly altered metabolites. Metabolites with adjusted $p$-value $<0.05$ and $\mid \log 2$ (fold change) $\mid>1$ were considered significant change. The heatmap was plotted using R package Complex Heatmap.

\section{DNA extraction and qPCR of microbiota in mouse stool}

Total DNA was extracted from aliquots of $100 \mathrm{mg}$ of mouse stool. Markedly altered bacteria were evaluated with quantitative PCR (qPCR) analyses, as previously described [16].

\section{Statistical analysis}

All statistical tests were performed using SPSS or GraphPad Software. Data are presented as mean \pm SD. Cell viability assay was analyzed with repeated-measures ANOVA. Comparison between two groups was analyzed with Paired twotailed Student's $t$ tests.

\section{Results}

\section{Curcumin sensitizes response to Ara- $C$ in xenograft model but not in AML cells}

To address the synergistic effect between Curcumin and Ara-C, HL-60 cells were xenografted into male NSGimmunodeficient mice by tail vein as previously described. At week 3 after HL-60 cells injection, the symptoms of disease appeared. After the establishment of disease, mice were treated with either Curcumin + Ara-C (Cur/A) or $\mathrm{PBS}+$ Ara-C (PBS/A) for 5 days consecutively. BM samples were collected on day 8 and monoclonal cells (MNCs) were isolated (Fig. 1A). CCK-8 assay showed that MNCs isolated from Cur/A group displayed significantly reduced cell proliferation rate compared to PBS/A group (Fig. 1B). Apoptosis assay also revealed that Cur/A led to markedly increase of MNCs compared to the PBS/A group (Fig. 1C). To further confirm this observation, we performed in vitro assay that HL-60 and THP-1 cells were treated with serial concentrations of Curcumin $(100,50,25,12,6,3,1$ and $0 \mu \mathrm{M})$ and Ara-C (3 $\mu \mathrm{M})$ (Cur/A or PBS/A treatment). Inconsistent with what observed in the animal model, we did not see significant alteration in Cur/Ara co-treated group compared to PBS/A-treated group as evidenced by CCK- 8 and apoptosis assay (Fig S1 A-D).

\section{Curcumin leads to altered intestinal microbiota}

Due to the inconsistence between the in vivo and in vitro analysis, we asked whether this inconsistence is associated with intestinal microbiota which have been established relationship with AML [17]. To address this question, we first compared the microbiota profiling between Cur/A-treated 
A

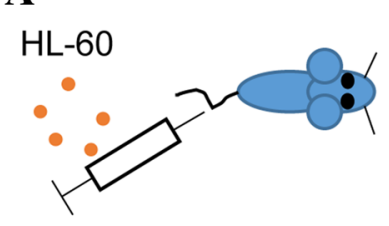

B

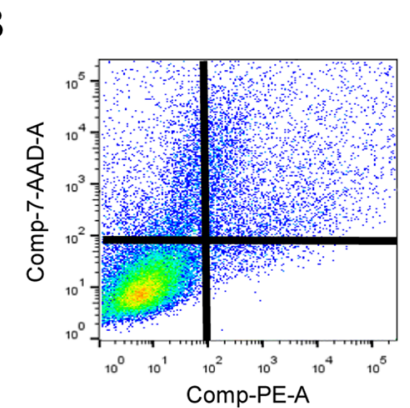

Cur/A

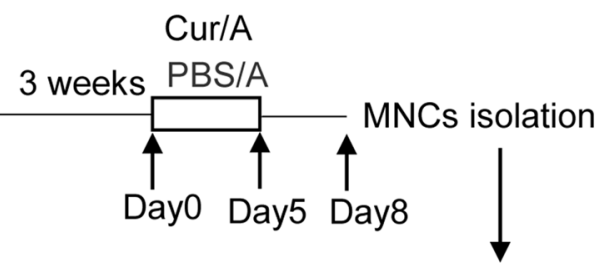

Proliferation and apoptosis analysis
C

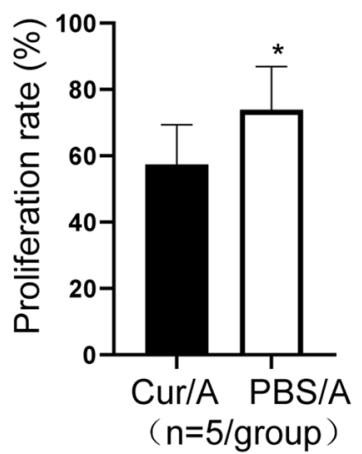

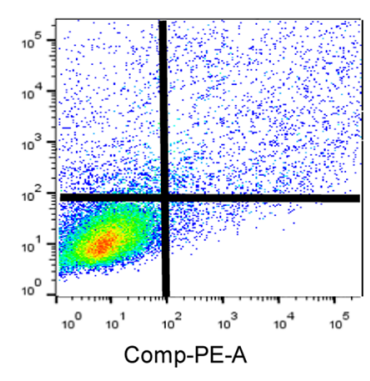

$\mathrm{PBS} / \mathrm{A}$

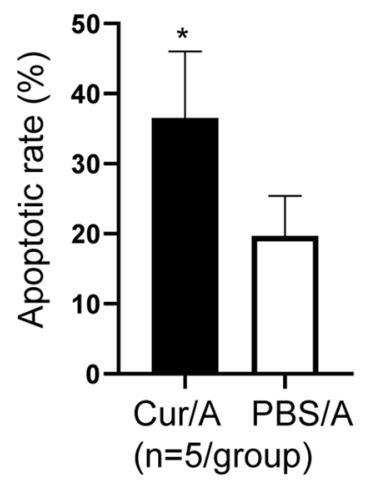

Fig. 1 Curcumin and Ara-C displayed synergistic effect in mice but not in cell lines. A Establishment of AML mouse model and experimental design. B, C MNCs isolated from Cur/A AML mice showed

mice and PBS/A-treated mice. $\alpha$-diversity analysis revealed an unchanged enrichment of bacteria species as determined by Shannon index and Chao-1 (Fig. 2A). However, principal component analysis (PCA) based on species-level revealed a significant alteration of intestinal microbiota composition between Cur/A and PBS/A group (Fig. 2B). Heatmap showed that Lactobacillus Acidophilus (L. acidophilus), Bifidobacterium bifidum (B. bifidum) and Lactobacillus reuteri ( $L$. reuteri) were significantly increased while pathologic bacteria, including Bacteroides fragilis (B. fragilis), Escherichia coli (E. Coli), Fusobacterium nucleatum (Fn) and Akkermansia muciniphila (A. muciniphila) were significantly reduced (Fig. 2C and Table S1). These observations indicate that Curcumin combined with Ara-C is associated with a different microbiota composition compared to Ara-C alone.

\section{Altered intestinal microbiota is involved in Curcumin-mediated increased response to Ara-C}

To determine whether altered microbiota induced by Curcumin is associated with sensitized response to Ara-C, we used germ free mice (GF) to develop AML xenograft model, followed by Curcumin and Ara-C treatment as mentioned above. Then MNCs were isolated for viability and significantly increased apoptosis in concomitant with decreased cell proliferation. ${ }^{*} p<0.05$

apoptosis assay (Fig. 3A). The time of the appearance of disease symptoms in GF mice was similar with the SPF mice. Interestingly, the synergistic effect between Curcumin and Ara-C disappeared in GF mice (Fig. 3B), suggesting Curcumin regulate microbiota to enhance response to Ara-C in AML. To further confirm this observation, GF mice were treated with stools of Cru/A-treated mice or stools of PBS/A mice (Fig. 3C). To confirm the colonization of bacteria, we checked the volume of altered bacteria using qPCR. It showed that $B$.fragilis, E. Coli and $F n$ were significantly reduced in Cur/A-treated-mice-stool-gavaged mice in concomitant with decreased B. fragilis, E. Coli, $F n$ and A.muciniphila, indicating a successful colonization (Fig. S2). Therefore, we treated the mice with Ara-C. Consistently, Cur/A-treated-mice-stool-gavaged mice displayed significantly reduced viability in concomitant with increased apoptosis in MNCs (Fig. 3D). These results indicate that Curcumin regulates intestinal microbiota to sensitize response to Ara-C.

\section{Curcumin enhances intestinal intact to sensitize response to $A M L$}

Intestinal intact is important in separating intestinal pathogen from epithelial cells to avoid pathogen invasion [18]. 
A

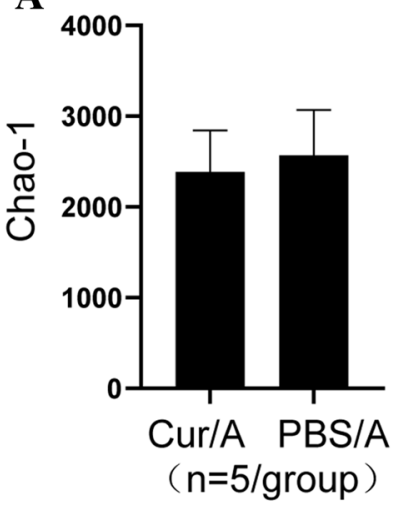

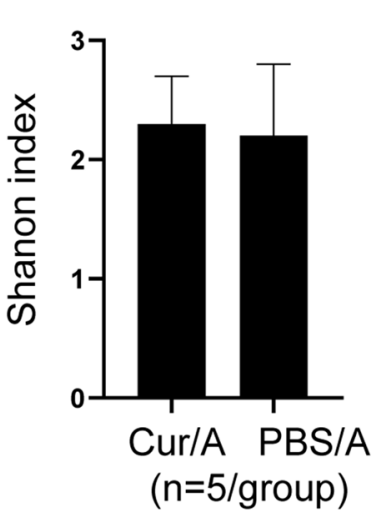

Ara-C
Cur/A

\author{
C
}

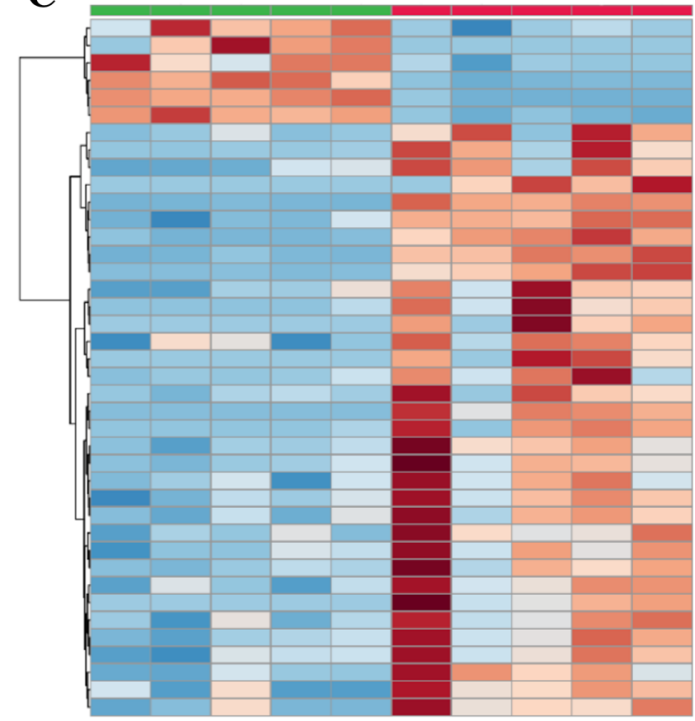

Ezakiella massiliensis Rhizobium sp. Y9
Caldicellulosiruptor bes Bactercides fragilis
Lactobacillus reuteri Lactobacillus reuteri Kerstersia gyiorum Ectothiorhodospira sp. BSL-9
Pseudomoss sp DR 5-09 Niaudella koreensis
Nis. DR Akkermansia muciniphi Vitivalales bacterium CCUG 44730 Escherichia coli
Bacteroides fragilis Eacteroides fragilis
Fusobacterium nucleatum Bordetella genomosp. 6
Bordetella sp. N Streptomyces sp. P3
Phytobacter ursingii Phytobacter ursingii
Legionella longbeach Halotalea alkalilenta Lelliotitia amnigeo Virgibacillus haloden Moraxella catarrhalis Actinobacillus porcitonsillarum
Actinobacteria bacterium YIM 96077 Aeromoos encheleia
Breoghania sp. L-A4 Breoghania Sp. L-A4
Escherichia sp. E4742 Marinobacter hydrocarbonoclasticas Gordonia Sp. MMS17-SY073 Streptomyces sp. P3
Oxalobacter formigenes Paenibacillus sp. FSL P4-008 Hydrogenobacter thermophilus Nocardiopsis dassonvillei
Microcella alkaliphila

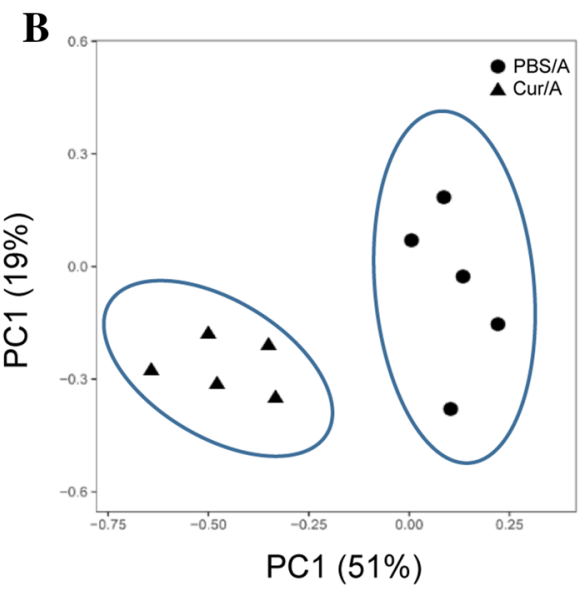

Fig. 2 Curcumin treatment led to intestinal microbiota alteration. A $\alpha$-diversity showed no significant change of bacteria diversity alteration with curcumin treatment as evidenced by Chao-1 and Shannon index analysis. B PCA assay revealed that Curcumin treatment

Mucus and tight junction proteins (TJPs) are key component to maintain intestinal intact [19]. As we observed the reduction of bacteria with the function of degrading intestinal mucus in Curcumin-treated mice, we asked whether Curcumin could strengthen intestinal intact. To address this question, FITC-dextran was administrated to mice $4 \mathrm{~h}$ before killing. Then, blood was collected for FITC determination. Consistent with our hypothesis, the concentration of FITCdextran was significantly reduced in serum of Cur/A-treated mice (Fig. 4A). Tight junction proteins ZO-1, occludin and claudin-1 were also increased in Cur/A-treated mice (Fig. 4B). Next, we investigated whether enhanced intestinal intact could sensitize response to Ara-C in AML. Probiotics (VSL\#3) was previously reported to strengthen intestinal intact. AML mice were treated with VSL\#3 (15 mg in 200ul PBS per mouse and treated to mice one week before resulted in markedly altered microbiota composition. C Heatmap showed that Curcumin induced probiotics enrichment while pathogenic bacteria were reduced

Ara-c treatment) plus Ara-C (VSL/A) or PBS/A (Fig. 4C). It turned out that MNCs of VSL/A-treated AML mice showed significantly decreased proliferation rate and increased apoptosis compared to PBS/A-treated AML mice (Fig. 4D). These results indicate that Curcumin enhances response to Ara-C by strengthening intestinal intact.

\section{Curcumin-induced microbiota alteration affects intracellular cholesterol synthesis}

Cellular metabolites were proved to be associated with chemoresistance [20]. To disclose the mechanism of Curcumin sensitizing response to Ara-C, we performed metabolomics for BM MNCs collected from Cur/A- and PBS/A-treated mice. The composition of metabolites in MNCs of the two groups of AML mice was significantly 


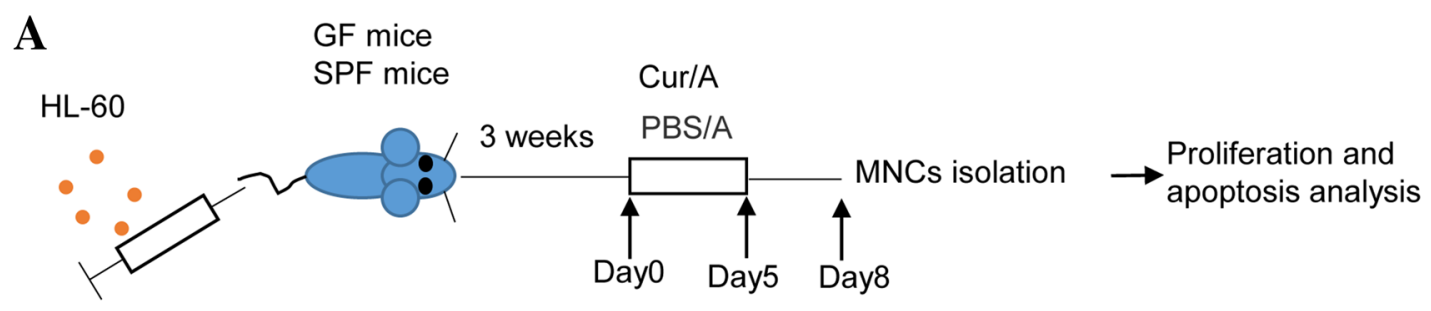

B
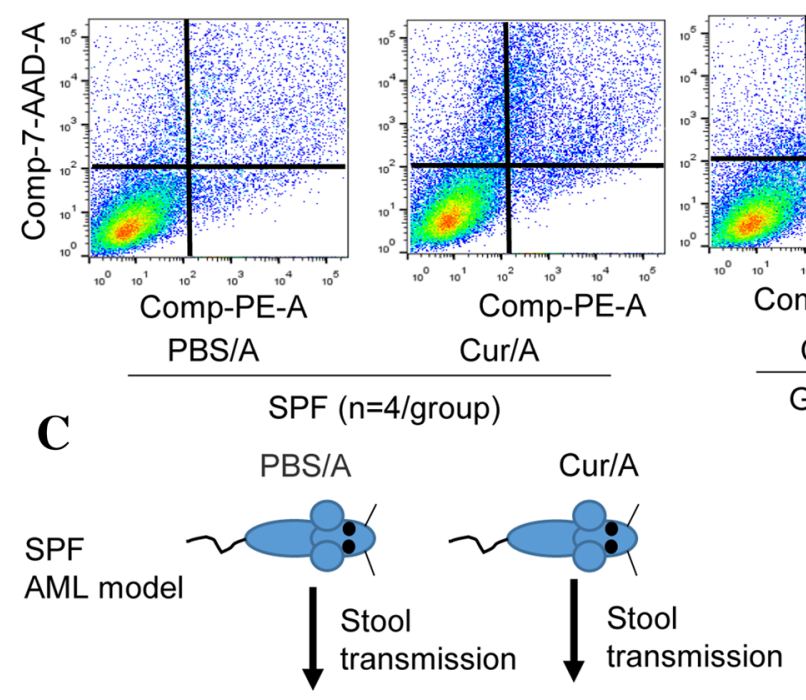

GF

AML model

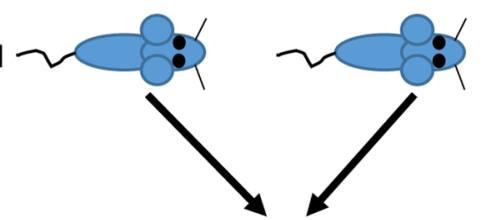

Ara-C treatment

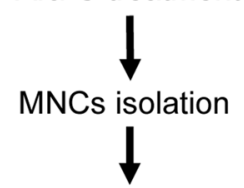

D
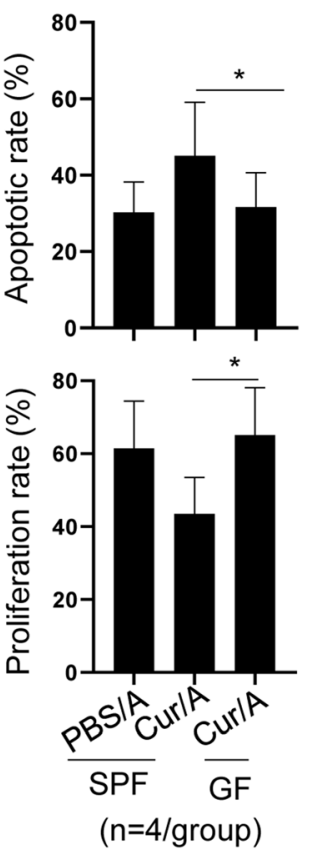

Proliferation and apoptosis analysis
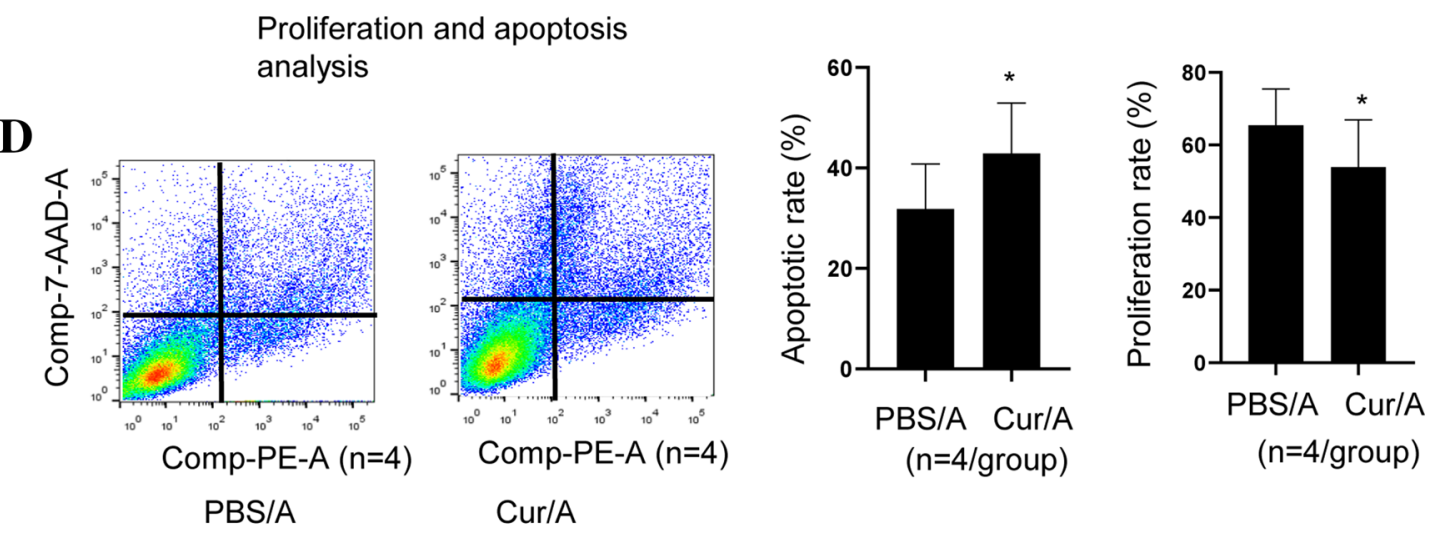

GF $(n=4)$ 
A

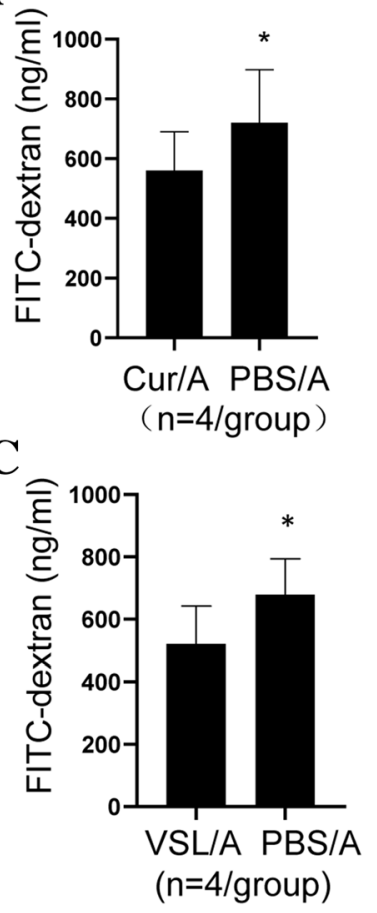

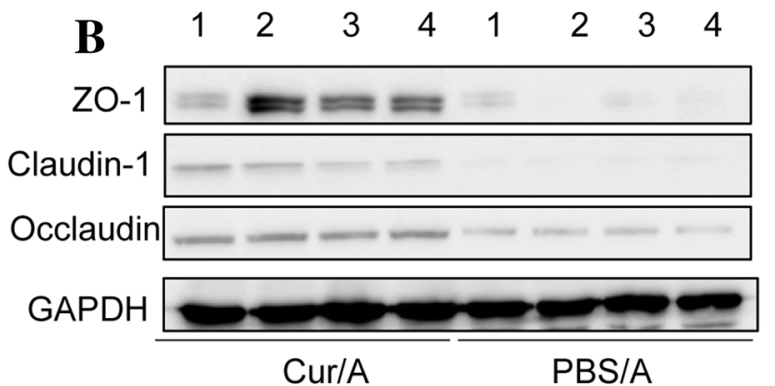

D GF mice
SPF mice

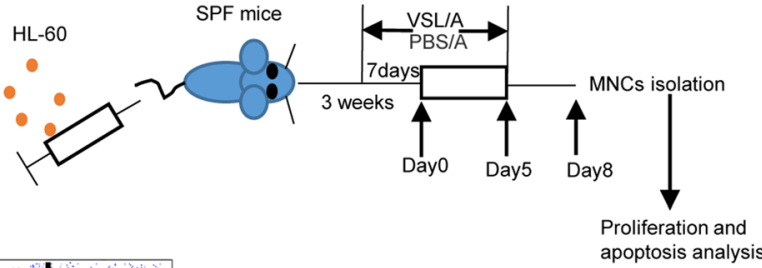

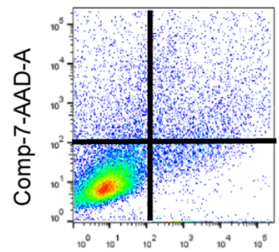

PBS/A

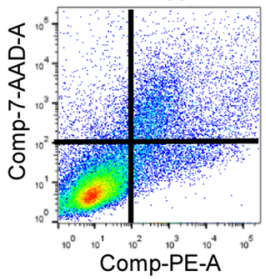

VSL/A
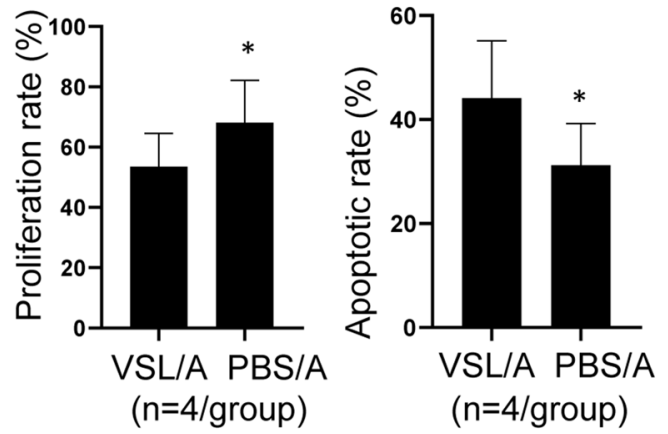

Fig. 4 Curcumin treatment enhanced intestinal intact. A Fitc-Dextran assay showed that Curcumin treatment led to decreased intestinal permeability. B TJPs, ZO-1, Occludin and Claudin-1, were up-regulated in Cur/A-treated mice. C Probiotics treatment reduced intestinal per- meability compared to control group. D Apoptosis and proliferation assay showed significantly increased apoptosis and decreased proliferation rate in MNCs of probiotics-treated AML mice. ${ }^{*} p<0.05$

L. acidophilus and B. bifidum were negatively correlated with cholesterol (Fig. 5E). These observations indicate that Curcumin-induced microbiota alteration reduced intracellular cholesterol level of MNCs.

\section{Curcumin-induced microbiota sensitizes response to Ara-C by suppressing SQLE}

It was reported that cholesterol accumulation was associated with chemoresistance [21]. On the other hand, we noticed that Squalene acts as the substrate of Squalene Epoxidase (SQLE) for cholesterol biosynthesis. So, we hypothesized that SQLE is involved in the Curcumin-mediated sensitized response to Ara-C in AML. WB showed that SQLE expression was inhibited in MNCs of Cur/A-treated AML mice
BS/A-treated mice. It showed that A. muciniphila an

E.Coli were positively correlated with cholesterol, whereas 


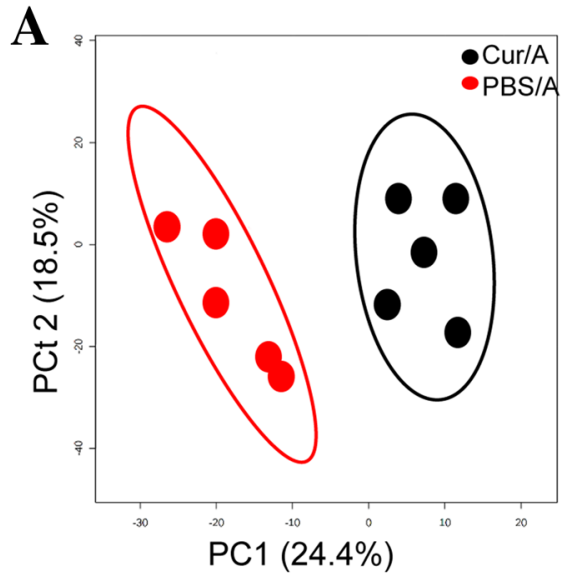

B

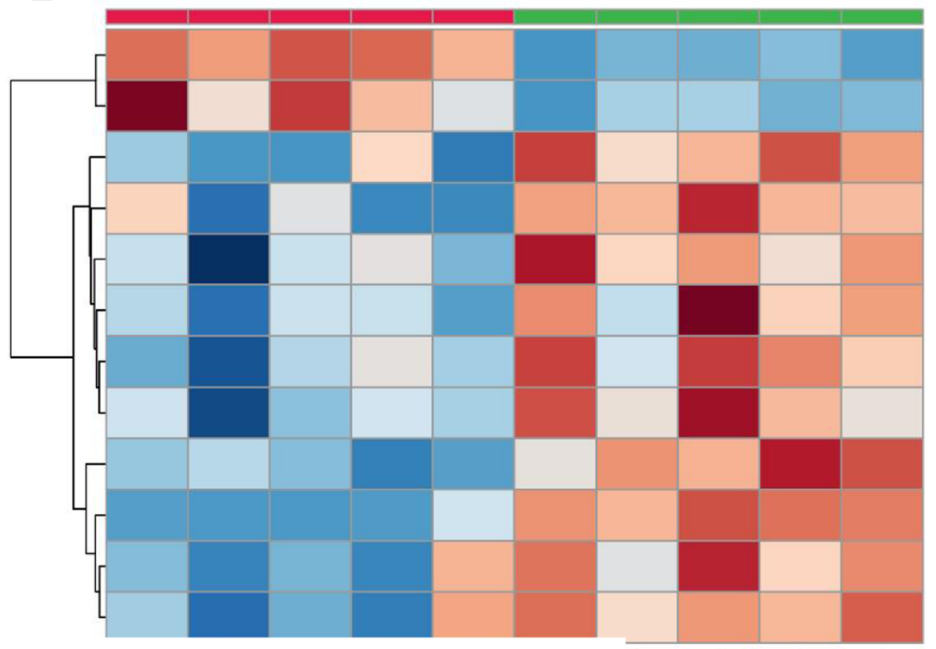

$\mathbf{E}$
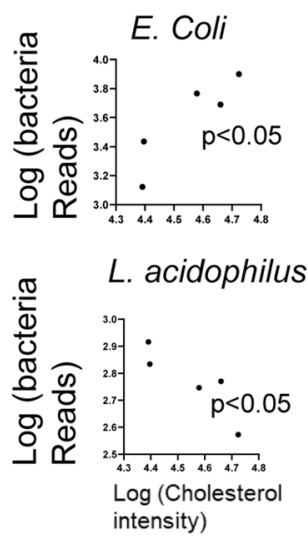

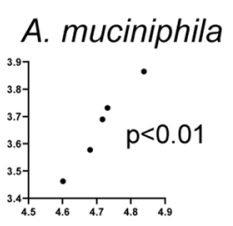

B. bifidum

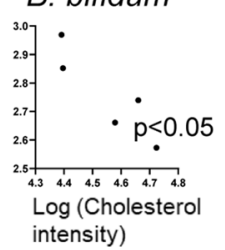

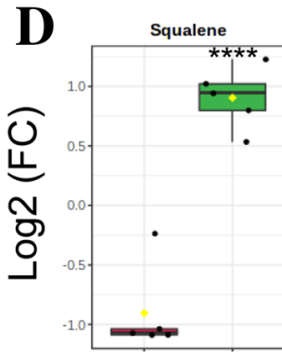

PBS/A Cur/A

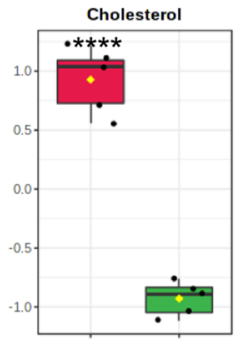

PBS/A Cur/A
Fig. 5 Curcumin-induced microbiota alteration led to change of metabolites in MNCs of AML mice. A PCA analysis saw an altered metabolomics of MNCs between Cur/A- and PBS/A-treated AML mice. B Heatmap showed markedly altered metabolites. C Volcano plot revealed that cholesterol and squalene were located at the outlier of significantly changed metabolites. D Curcumin treatment led to significantly down-regulated cholesterol in MNCs of AML mice. $* * p<0.01, * * * * p<0.0001$ 


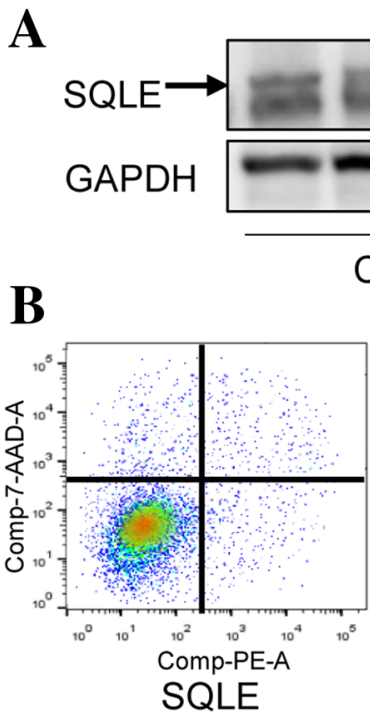

C

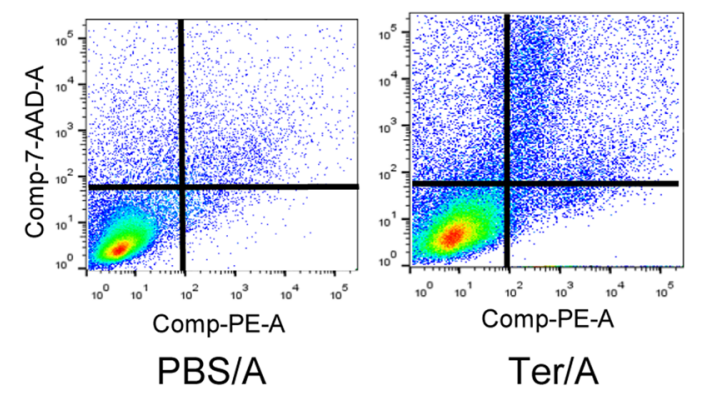

Fig. 6 Curcumin sensitized response to Ara-C through suppressing SQLE. A SQLE was up-regulated in MNCs of Cur/A-treated AML mice. B SQLE over-expression in THP-1 cells led to markedly

compared to MNCs of PBS/A-treated mice (Fig. 6A) To further confirm the function of SQLE, THP-1 cells were transfected with SQLE plasmids. As a result, SQLE overexpression led to a significant reduction of cell apoptosis as well as increased cell viability (Fig. 6B). To further assess the function of SQLE we used Terbinafine, a selective SQLE inhibitor, to treat AML mice. Similar with what observed in cells, SQLE inhibition resulted in sensitized response to Ara-C in MNCs of AML mice (Fig. 6C). These results suggest that Curcumin sensitizes response to Ara-C in AML by suppressing SQLE.

\section{Discussion}

In the present study, we investigated the effect of Curcumin on the response to Ara-C. A xenograft AML mouse model was established to assess the synergistic effect
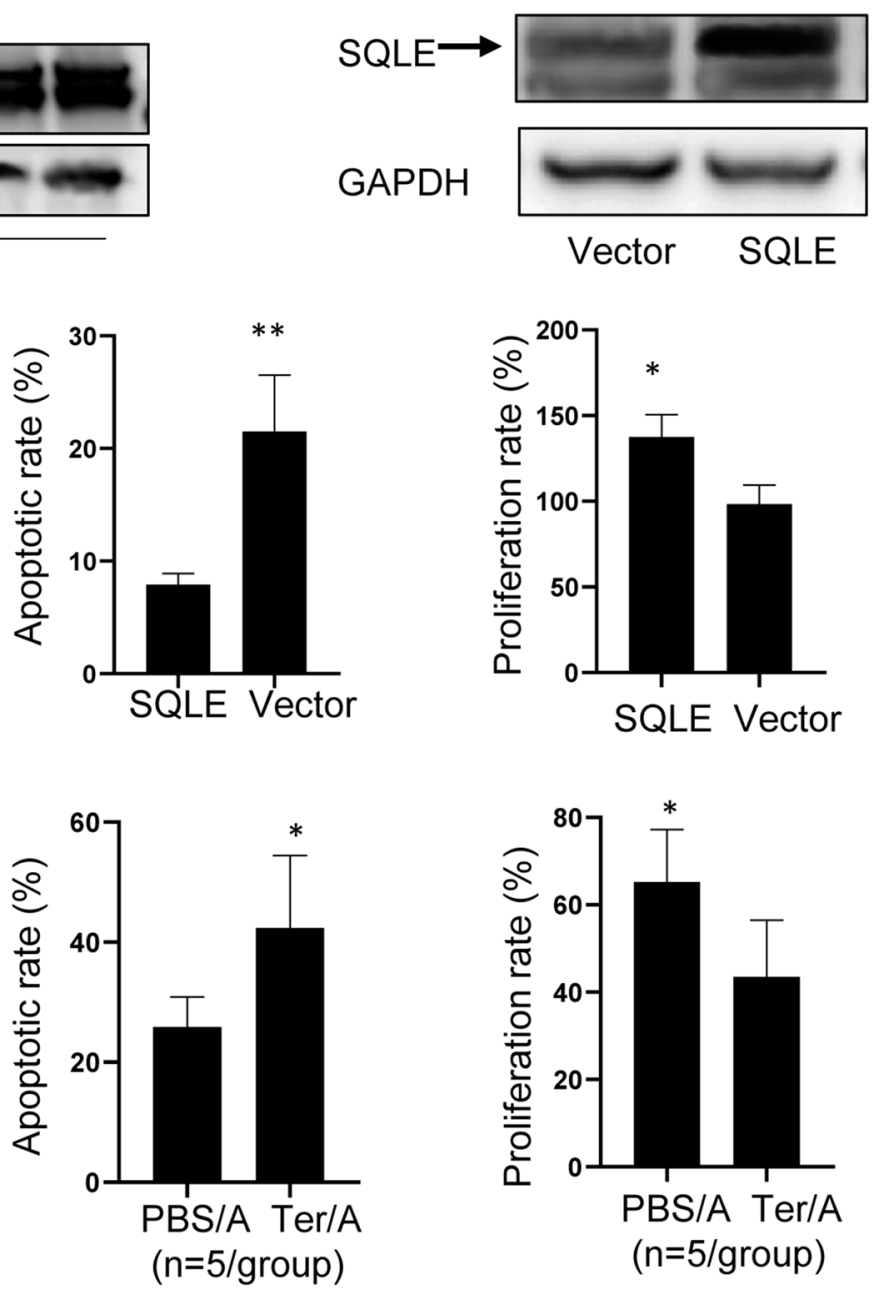

decreased apoptosis as well as increased proliferation. C SQLE inhibitor resulted in significantly increased apoptosis and reduced proliferation in MNCs of AML mice. ${ }^{*} p<0.05$, ** $p<0.01$

between Curcumin and Ara-C. We found that Curcumin, unlike the observations in other disease, could not directly sensitize response to Ara-C as we did not see a synergistic effect between Curcumin and Ara-C in the in vitro analysis. Instead, it regulates intestinal microbiota to sensitize response to Ara-C. In the metagenomic result, we found that several bacteria which were identified as probiotics, such as L. Acidophilus, B. bifidum and L. reuteri were enriched by Curcumin. These bacteria protected intestine through inhibiting inflammation. In contrast, some pathogenic bacteria were decreased, including E.Coli, A.Muciniphila and B.fragilis. E.Coli and B.fragilis were demonstrated to cooperate in invading into deep layer of intestine [22]. A.Muciniphila was proved to degrade intestinal mucus to break its intact [23]. In intestine related disease, the break of intestinal mucus was considered to be the initial step of disease, allowing the invasion of bacteria to cause inflammation. Intestinal inflammation allows the 
dysregulation of TJPs, allowing the intestinal pathogen to invade into deep place of intestine [18]. Here, we also found that Curcumin treatment led to ameliorated TJPs dysregulation, which may be associated with the enriched probiotics. Recently, a study revealed that strengthening intestinal barrier is beneficial for AML chemotherapy through reduction of bacterial translocation [17]. On the other hand, study reported that substantial bacteria was also detected in other cancerous tissues [24], indicating intestinal bacteria can transfer from intestine to other tissues. In our study, we also found that strengthening intestinal intact sensitized response to Ara-C, which was associated with suppressed SQLE induced by Curcuminmediated microbiota alteration. However, we did not uncover how SQLE was suppressed. Based on the current findings, the possibility of Curcumin-mediated sensitized response to Ara-C is: Curcumin resulted in strengthening intestinal intact, leading to reduced transfer of bacteria (or their metabolites) to the blood, causing SQLE inhibition. Moreover, a positive correlation between intestinal intact breaking bacteria E.Coli and A.Muciniphila and cholesterol in MNCs further indicating that up-regulation of cholesterol is associated with intestinal break. As we all know, SQLE is a key enzyme in cholesterol biosynthesis, which has been reported to be oncogenic in several disease $[25,26]$. Our study fulfills a further recognition of SQLE in AML.

In conclusion, we demonstrated that Curcumin sensitizes response to Ara-C by regulating microbiota and strengthening intestinal intact is promising in chemoresistant therapy. Moreover, controlling intracellular cholesterol level may be an effective way to ameliorate chemoresistance.

Supplementary Information The online version contains supplementary material available at https://doi.org/10.1007/s00280-021-04385-0.

Acknowledgements We thank the Animal Center of Shenzhen Longhua New District people's Hospital for their help in AML xenograft mouse model establishment. We also thank Novogene for the metagenomics and metabolomics analysis.

Author contributions JL: designed and supervised the project, data analysis and modified manuscript. WL: AML mice establishment and drafted manuscript. QC: cell experiments and data analysis. XC: western blotting and animal experiments. GZ: apoptosis and proliferation assay. HS: language modification.

Funding This study was funded by Shenzhen Fundamental Research Program (No. JCY2018022864237514).

Data availability The datasets generated during and/or analyzed during the current study are not publicly available due to the on-going related projects but are available from the corresponding author on reasonable request.
Code availability N/A.

\section{Declarations}

Conflict of interest The authors declare that they have no conflict of interest.

Ethics approval The animal study was approved by the committee of Shenzhen Longhua New District People's Hospital. All animal experiments comply with the ARRIVE guidelines and carried out in accordance with the National Institutes of Health guide for the care and use of Laboratory animals.

\section{Consent to participate N/A.}

Consent for publication N/A.

Open Access This article is licensed under a Creative Commons Attribution 4.0 International License, which permits use, sharing, adaptation, distribution and reproduction in any medium or format, as long as you give appropriate credit to the original author(s) and the source, provide a link to the Creative Commons licence, and indicate if changes were made. The images or other third party material in this article are included in the article's Creative Commons licence, unless indicated otherwise in a credit line to the material. If material is not included in the article's Creative Commons licence and your intended use is not permitted by statutory regulation or exceeds the permitted use, you will need to obtain permission directly from the copyright holder. To view a copy of this licence, visit http://creativecommons.org/licenses/by/4.0/.

\section{References}

1. Ferrara F, Schiffer CA (2013) Acute myeloid leukaemia in adults. Lancet 381:484-495. https://doi.org/10.1016/S0140-6736(12) 61727-9

2. Li Y, Shao H, Da Z et al (2019) High expression of SLC38A1 predicts poor prognosis in patients with de novo acute myeloid leukemia. J Cell Physiol 234:20322-20328. https://doi.org/10. $1002 / j \mathrm{cp} .28632$

3. Wilken R, Veena MS, Wang MB et al (2011) Curcumin: A review of anti-cancer properties and therapeutic activity in head and neck squamous cell carcinoma. Mol Cancer 10:12. https://doi.org/10. 1186/1476-4598-10-12

4. MJ Xavier, GM Dardengo, C Navarro-Guillen et al (2021) Dietary Curcumin Promotes Gilthead Seabream Larvae Digestive Capacity and Modulates Oxidative Status. Animals (Basel) 11.

5. Bateni Z, Rahimi HR, Hedayati M et al (2012) The effects of nano-curcumin supplementation on glycemic control, blood pressure, lipid profile, and insulin resistance in patients with the metabolic syndrome: a randomized, double-blind clinical trial. Phytother Res 35:3945-3953. https://doi.org/10.1002/ptr.7109

6. Xu X, Wang H, Guo D et al (2021) Curcumin modulates gut microbiota and improves renal function in rats with uric acid nephropathy. Ren Fail 43:1063-1075. https://doi.org/10.1080/ 0886022X.2021.1944875

7. C Cheng, F Yuan, XP Chen et al (2021) Inhibition of Nrf2-mediated glucose metabolism by brusatol synergistically sensitizes acute myeloid leukemia to Ara-C. Biomed Pharmacother 111652. https://doi.org/10.1016/j.biopha.2021.111652, 111652

8. Z Han, L Yao, Y Zhong et al (2015) Gut microbiota mediates the effects of curcumin on enhancing Ucp1-dependent thermogenesis 
and improving high-fat diet-induced obesity. Food Funct, 2021. https://doi.org/10.1039/d1fo00671a.

9. Saland E, Boutzen H, Castellano R et al (2015) A robust and rapid xenograft model to assess efficacy of chemotherapeutic agents for human acute myeloid leukemia. Blood Cancer J 5:e297. https:// doi.org/10.1038/bcj.2015.19

10. M Hosseini, HR. Rezvani, N Aroua et al (2019) Targeting Myeloperoxidase Disrupts Mitochondrial Redox Balance and Overcomes Cytarabine Resistance in Human Acute Myeloid Leukemia. Cancer Res 79: 5191-5203. https://doi.org/10.1158/0008-5472. CAN-19-0515.

11. Abraham A, Krasnodembskaya A (2020) Mesenchymal stem cellderived extracellular vesicles for the treatment of acute respiratory distress syndrome. Stem Cells Transl Med 9:28-38. https://doi. org/10.1002/sctm.19-0205

12. Gupta J, del Barco Barrantes I, Igea A et al (2014) Dual function of p38alpha MAPK in colon cancer: suppression of colitis-associated tumor initiation but requirement for cancer cell survival. Cancer Cell 2014(25):484-500. https://doi.org/10.1016/j.ccr. 2014.02.019

13. Li H, Durbin R (2010) Fast and accurate long-read alignment with Burrows-Wheeler transform. Bioinformatics 26:589-595. https:// doi.org/10.1093/bioinformatics/btp698

14. Li H, Handsaker B, Wysoker A et al (2009) The Sequence Alignment/Map format and SAMtools. Bioinformatics 25:2078-2079. https://doi.org/10.1093/bioinformatics/btp352

15. Wood DE, Lu J, Langmead B (2019) Improved metagenomic analysis with Kraken 2. Genome Biol 20:257. https://doi.org/10. 1186/s13059-019-1891-0

16. Godon JJ, Zumstein E, Dabert P et al (1997) Molecular microbial diversity of an anaerobic digestor as determined by small-subunit rDNA sequence analysis. Appl Environ Microbiol 67:2802-2813. https://doi.org/10.1128/aem.63.7.2802-2813.1997

17. Hueso T, Ekpe K, Mayeur $\mathrm{C}$ et al (2020) Impact and consequences of intensive chemotherapy on intestinal barrier and microbiota in acute myeloid leukemia: the role of mucosal strengthening. Gut Microbes 12:1800897. https://doi.org/10.1080/19490976.2020. 1800897

18. Corazziari ES (2009) Intestinal mucus barrier in normal and inflamed colon. J Pediatr Gastroenterol Nutr 48(Suppl 2):S54S55. https://doi.org/10.1136/gut.2006.098160
19. Swidsinski A, Loening-Baucke V, Theissig F et al (2007) Comparative study of the intestinal mucus barrier in normal and inflamed colon. Gut 56:343-350. https://doi.org/10.1136/gut.2006.098160

20. Hanahan D, Weinberg RA (2011) Hallmarks of cancer: the next generation. Cell 144:646-674. https://doi.org/10.1016/j.cell.2011. 02.013

21. Mayengbam SS, Singh A, Pillai AD et al (2021) Influence of cholesterol on cancer progression and therapy. Transl Oncol 14(101043):x. https://doi.org/10.1016/j.tranon.2021.101043

22. Dejea CM, Fathi P, Craig JM et al (2018) Patients with familial adenomatous polyposis harbor colonic biofilms containing tumorigenic bacteria. Science 359:592-597. https://doi.org/10.1126/ science.aah3648

23. Yoshihara T, Oikawa Y, Kato T et al (2020) The protective effect of Bifidobacterium bifidum G9-1 against mucus degradation by Akkermansia muciniphila following small intestine injury caused by a proton pump inhibitor and aspirin. Gut Microbes 11:13851404. https://doi.org/10.1080/19490976.2020.1758290

24. Nejman D, Livyatan I, Fuks G et al (2020) The human tumor microbiome is composed of tumor type-specific intracellular bacteria. Science 368:973-980. https://doi.org/10.1126/science.aay91 89

25. L He, H Li, C Pan et al (2021) Squalene epoxidase promotes colorectal cancer cell proliferation through accumulating calcitriol and activating CYP24A1-mediated MAPK signaling. Cancer Commun (Lond) 2021. https://doi.org/10.1002/cac2.12187.

26. EB. Zhang, X Zhang, K Wang et al (2021) Antifungal agent Terbinafine restrains tumor growth in preclinical models of hepatocellular carcinoma via AMPK-mTOR axis. Oncogene 2021. https:// doi.org/10.1038/s41388-021-01934-y.

Publisher's Note Springer Nature remains neutral with regard to jurisdictional claims in published maps and institutional affiliations. 